AperTO - Archivio Istituzionale Open Access dell'Università di Torino

\title{
Quantified Self and Modeling of Human Cognition
}

\section{This is the author's manuscript}

Original Citation:

\section{Availability:}

This version is available http://hdl.handle.net/2318/1577113

since 2016-06-30T18:40:37Z

Publisher:

ACM

Published version:

DOI:10.1145/2800835.2800954

Terms of use:

Open Access

Anyone can freely access the full text of works made available as "Open Access". Works made available under a Creative Commons license can be used according to the terms and conditions of said license. Use of all other works requires consent of the right holder (author or publisher) if not exempted from copyright protection by the applicable law. 
This is the author's final version of the contribution published as:

Cena, F.; Likavec, S.; Rapp. A.. Quantified Self and Modeling of Human Cognition, in: Adjunct Proceedings of the 2015 ACM International Joint Conference on Pervasive and Ubiquitous Computing and Proceedings of the 2015 ACM International Symposium on Wearable Computers (UbiComp/ISWC'15 Adjunct), ACM, 2015, 978-1-4503-3575-1, pp: 1021-1026.

The publisher's version is available at: http://dl.acm.org/citation.cfm?doid=2800835.2800954

When citing, please refer to the published version.

Link to this full text:

http://hdl.handle.net/2318/1577113 


\section{Quantified Self and Modeling of Human Cognition}

\author{
Federica Cena \\ University of Torino \\ Torino, 10149, Italy \\ cena@di.unito.it

\section{Silvia Likavec} \\ University of Torino \\ Torino, 10149, Italy \\ likavec@di.unito.it \\ Amon Rapp \\ University of Torino \\ Torino, 10149, Italy \\ amon.rapp@gmail.com
}

Paste the appropriate copyright/license statement here. ACM now supports three different publication options:

- ACM copyright: ACM holds the copyright on the work. This is the historical approach.

- License: The author(s) retain copyright, but ACM receives an exclusive publication license.

exclusive publication license. Open Access: The author(s) wish to pay for the
access. The additional fee must be paid to ACM.

This text field is large enough to hold the appropriate release statement assuming it is single-spaced in Verdana 7 point font. Please do not change the size of this text box.

Each submission will be assigned a unique DOI string to be included here.

\begin{abstract}
This paper tackles an important issue of how to use quantified self technologies for modeling human cognition. At the same time, it provides some insights on how Quantified Self community can benefit from user modeling and personalization, especially in the domain of human cognition.
\end{abstract}

\section{Author Keywords}

Quantified Self; personal informatics; user modeling; cognitive modeling; cognitive assessment.

\section{ACM Classification Keywords}

H.5.m. Information interfaces and presentation (e.g., $\mathrm{HCI}$ ): Miscellaneous.

\section{Introduction}

Personalized systems tailor the system response to the preferences and needs of each specific user, stored in the User Model (UM) [3]. Traditional UMs usually contain user demographic data (e.g., gender, age, etc.) explicitly provided by users and domain-specific data (e.g. interest in certain topics) provided by implicitly observing user behavior on the web [10] or by collecting user information provided by social networking sites via APIs [16]. 
Quantified Self (QS) is a new trend which helps people acquire relevant personal information on different aspects of their lives, with the purpose of selfmonitoring [13]. The technologies that support data collection, aggregation and visualization by means of computerized graphics are called Personal Informatics (PI) tools. They can be apps running on users' mobile devices (such as Moves for automatic tracking of steps or Dreamboard for collection of dreams) or they can be ad hoc smart wearable devices (such as Jawbone UP).The diffusion of PI tools [12] opens up new opportunities for user modeling. The advancements of wearable and ubiquitous technologies allow for an automatic detection of a variety of data: physiological states (e.g. blood pressure), cognitive states (e.g. stress), behavior (e.g. movements or average number of hours sleeping), spatial context (e.g. places visited) and social context (e.g. people met or interacted with). This means that PI tools can nurture the UM with a plethora of data gathered from user's actions and behavior in real life and not limited to the user's web activity, as usually happens in traditional UMs [3].

All the obtained data about the users' real life behavior can be used for the creation and management of long term, even lifelong, user models. These UMs capture salient aspects about the user over very long periods of time and handle changing interests over time (Lifelong user modeling) [9]. Our aim is to build on top this type of UMs and exploit the possibilities offered by PI tools not only for modeling interests, goals, values and preferences, but also human behavior, emotions and cognition. Hence, we propose to design an Enhanced User Model (EUM) containing four types of data, each of which contains short-term and long-term information:
- user attitudes: opinions and preferences w.r.t. to a specific object or event, or believes and tastes w.r.t. a trend in time;

- user behaviors: actions in real world happening in an exact time (such as movements and tasks), or habits (recurrent and repetitive sequence of actions);

- user affects: emotions (when spanning a short period of time), or mood (emotional trends that span a longer period of time);

- user cognitive functions: cognitive states (e.g. memory, level of attention) regarding a specific moment, and cognitive skills regarding their level of functioning in time, such as their improvement or decline (e.g. memory health, or decline of spatial orientation due to a pathological drift).

In the following we will focus on the part of EUM related to the user's cognitive functions, which we intend to infer from the data coming from real world behavior and made available by the current PI technologies. Moreover, we explore a possible impact this EUM has on the QS world. Our innovative approach to user modeling offers new ways for management, integration and interpretation of personal information, by providing a complete digital image of the user. This can help users create a more comprehensive vision of themselves and improve multiple aspects of their lives.

\section{Related work}

User Model. Traditional user modeling is mostly used to enhance user's experience on the Web and make use of web-based information. In [14], the authors help the user search for information by adaptively selecting the most relevant items. Also, it is possible to provide adaptive navigation support by manipulating the links 
[4] or present the content of a Web page adaptively [5]. Recently, lifelong user models aim to collect personal information made available by pervasive and ubiquitous computing to both support personalization over the long term and serve long term goals [9]. Following this approach, we want to gather information about user's attitudes, behaviors, emotions and cognitive functions in the short and long term, enriching long term user models with data that come from the real world.

Cognitive User Model. Cognitive models have been seen by the literature as computer programs that can simulate human performance of cognitive skills [15] and can be used as surrogate users to help in user interface design. The user modeling approach in [7] is based on a cognitive theory of human memory which helps in defining the structures suitable for organizing the memory concepts and the algorithms for storage and retrieval of information. We intend this term differently, since we want to automatically recognize and assess the cognitive functions of a person, independently of the way they are organized.

Cognitive Quantified Self. This new trend shares with us the aim of obtaining a picture of user's cognitive states. Differently from us, authors in [11] monitor user cognitive behavior (e.g. reading) in order to infer user cognitive load and design augmented mind technologies. In our approach instead, starting from user's everyday activities in real world (walking, dressing, doing everyday tasks), we aim both to recognize and collect a specific cognitive state in a specific point in time and to assess the connected cognitive skill.
Cognitive Assessment. Traditionally, patient cognitive skills are assessed using various cognitive tests (e.g. Mini Mental State Examination or Clinical Dementia Rating) and observational scales (e.g. Bristol Activities of Daily Living Scale or Cohen Mansfield Agitation Inventory) [17] which help the physicians to determine patient's cognitive function levels and especially the level of its decline.

IoT-based Cognitive Assessment. Internet of Things technologies (i.e., sensors and cameras) [1] are recently being used to monitor specific user tasks in order to assess specific cognitive pathologies. For example, authors in [8] perform automatic assessment of cognitive impairment (dementia and traumatic brain injury) through electronic observation of objects usage. More specifically they monitor with sensors the task of making a coffee. Differently from us, these studies are conducted in a laboratory, and are very obtrusive for the user. In our opinion, the monitoring should be as transparent as possible. Other works only use environmental sensors to assess pathological states in real environment. For example, authors in [6] introduce a machine learning-based method to automatically predict activity quality in smart homes and automatically assess cognitive health. Differently from this family of works, we want to both collect single cognitive states (such as memories, degree of attention when performing a task, etc.) for the purpose of selfawareness and assess the related cognitive skill (e.g. memory, attention), for example for health benefits.

\section{Enriching the User Model by means of PI}

\section{tools}

We exploit PI tools to transparently and unobtrusively gather data about user's everyday behavior which we 
use to build the EUM. This data will be used to infer cognitive states. The main issue in this process is to overcame the gap between user's actions and her internal states. Our goals are: i) to recognize the different cognitive states of a person, such as memories, degrees of attention, degrees of orientation in a specific environment, capabilities to execute a plan; ii) to assess the level of functioning over time of the related cognitive functions, such as memory, attention, orientation, executive and praxis functions (i.e. the cognitive skills). The latter can be inferred by evaluating how the function is manifested in different occurrences over time. For each we maintain:

- functional indicators, i.e. activities and behaviors which can be considered as indicators of certain cognitive functions. These indicators are deduced from traditional observational scales and neuropsychological tests. For example, in several observational scales "wandering" is an indicator for "orientation", "coordination" is an indicator for "executive functions" and "apathy" is an indicator for "attention".

- operational definition, i.e. which exact activities or sequence of actions can be monitored by means of technological tools and how the results can be quantifiable. For example, it is possible to monitor wandering by analyzing the routes taken inside and/or outside the user's house.

- PI tools, i.e. which technologies (wearable and mobile devices) can be exploited for monitoring such activities, both automatically or by means of selfreporting. For example, we can decide to monitor "wandering" by using GPS on a mobile phone, and "coordination" by means of an accelerometer on a wearable device. Moreover, we can consider eyetracking while watching TV by means of a Kinect camera to measure, for example, the level of apathy.

So, by detecting the user's wandering in a specific moment in time, we can recognize the occurrence of the cognitive function of orientation; by inferring from the diverse occurrences over time, instead, we can assess the level of functioning of the cognitive function itself, i.e. the cognitive skill (e.g. the level of functioning of the individual's orientation can be inferred from its various actualizations in time, for example from recurrent episodes of wandering, allowing also to assess whether the user suffers of a decline of this function). The validation of the cognitive assessment through these technological means can be done by the comparison with the assessment resulting from traditional cognitive assessment tests, such as observational scales and neurological batteries [17].

\section{Enriching the QS with the EUM}

First, EUM will provide the quantified image of the user's self with a greater amount of user data (attitudes, behaviors, emotions, cognitive functions) and this will provide her with a complete picture of herself, from different points of view (cognitive, behavioral, etc). In particular, the cognitive part of EUM would allow users to have insights into their cognitive states and abilities inferred from their everyday behavior. Although some authors do monitor certain cognitive abilities directly (such as reading in [11]), we aim to recognize and track different cognitive functions simultaneously to compose a multifaceted representation of the user's mind, bringing insights into its functioning. 
Moreover, the data made available to the user from PI tools are usually visualizations of the monitored phenomena, with, at most, simple correlations among user's activities which are displayed using natural language [2]. EUM, and especially its cognitive part, would provide information one step ahead: it will allow to correlate not only the activities among themselves and with the user's attitudes, but also with the user's cognitive states and skills involved in those activities. For example, it would be possible to infer various attention levels for different tasks performed, probably depending on user's interest in those tasks and/or their subjects. In addition, continuously monitoring user's actions could reveal some habits that users are not aware of, or correlate them with cognitive states and emotions, when present. For example, even though the usual PI tools do allow users to become aware of some unconscious behavioral patterns (such as spending long time sitting), we want to provide reasons and motivation (such as detection of depressive thoughts and lack of exercise).

W.r.t. personalization we can point out two novel aspects. First, EUM might enable personalization of PI tools interface in order to better fit user needs and preferences, as well as cognitive functions. For example, the visualizations would have adequate level of detail respecting user's attention capabilities and content would be in sync with user's interests. Second, EUM would allow PI tools to provide specific

recommendations taking into account user's attitudes and cognitive functions.

\section{Conclusion}

In this work we presented EUM - Enhanced User Model, which is designed by employment of PI tools. It is envisioned as a step forward towards better understanding of human cognition and behavior. It also nourishes back the PI tools, providing users with complex images of themselves, offering opportunities for personal growth and change. We think that EUM can provide researchers with useful insights which can positively influence various fields. One of the directions where cognitive user modeling can be exploited is the ecological (seamless and unobtrusive) assessment of cognitive skills in elderly population for possible detection of early signals of cognitive decline and impairment. Subsequently, cognitive part of EUM can be used in developing personalized cognitive trainings addressing the specific declining cognitive skills and adapted to user's preferences. Also, EUM can be applied to collect data for assessing different types of cognitive skills and/or impairment in various populations (children, people with disability), proposing personalized interventions and trainings. Finally, EUM can found its application in video games or educational games for children.

\section{References}

1. Luigi Atzori, Antonio Iera, and Giacomo Morabito. 2010. The Internet of Things: A survey. Comput. Netw. 54, 15, 2787-2805.

2. Frank Bentley, Konrad Tollmar, Peter Stephenson, Laura Levy, Brian Jones, Scott Robertson, Ed Price, Richard Catrambone, and Jeff Wilson. 2013. Health Mashups: Presenting Statistical Patterns between Wellbeing Data and Context in Natural Language to Promote Behavior Change. ACM Trans. Comput.Hum. Interact. 20, 5, Article 30, 27 pages. http://doi.acm.org/10.1145/2503823

3. Peter Brusilovsky. 1996. Methods and techniques of adaptive hypermedia, User Modeling and UserAdapted Interaction, 6, 2-3, 87-129. 
4. Peter Brusilovsky. 2007. Adaptive navigation support. In The adaptive web, Peter Brusilovsky, Alfred Kobsa, and Wolfgang Nejdl (Eds.). LNCS 4321. Springer-Verlag, Berlin, Heidelberg 263-290.

5. Andrea Bunt, Giuseppe Carenini, and Cristina Conati. 2007. Adaptive content presentation for the web. In The Adaptive Web, Methods and Strategies of Web Personalization, Peter Brusilovsky, Alfred Kobsa, and Wolfgang Nejdl (Eds.). LNCS 4321. Springer-Verlag Berlin Heidelberg, 409-432.

6. Prafulla N. Dawadi, Diane J. Cook, Maureen Schmitter-Edgecombe, and Carolyn Parsey. 2013. Automated assessment of cognitive health using smart home technologies. Technol. Health Care 21 4, 323-343. http://dx.doi.org/10.3233/THC 130734

7. Fabio Gasparetti, and Alessandro Micarelli. 2008. A deep evaluation of two cognitive user models for personalized search. In Series in Machine Perception and Artificial Intelligence, Personalization Techniques and Recommender Systems, Gulden Uchyigit, Matthew Y. Ma (eds.), 70, World Scientific Publishing Co. 3-32.

8. Mark R. Hodges, Ned L. Kirsh, Mark W. Newman and Martha E. Pollack. 2010. Automatic Assessment of Cognitive Impairment through Electronic Observation of Object Usage. In Proceedings of the 8th International Conference on Pervasive computing (PERVASIVE 2010), 192-209. http://dx.doi.org/10.1007/978-3-642-12654-3_12

9. Judy Kay and Bob Kummerfeld. 2009. Lifelong User Modelling Goals, Issues and Challenges.

In Proceedings of the Lifelong User Modelling Workshop at UMAP 2009.

10. Alfred Kobsa, Jürgen Koenemann, and Wolfgang Pohl. 2001. Personalised hypermedia presentation techniques for improving online customer relationships. Knowl. Eng. Rev. 16, 2, 111-155.
11. Kai Kunze, Masai Katsutoshi, Yuji Uema, and Masahiko Inami. 2015. How much do you read?: counting the number of words a user reads using electrooculography. In Proceedings of the 6th Augmented Human International Conference $(\mathrm{AH}$ '15), 125-128.

http://doi.acm.org/10.1145/2735711.2735832

12. Ian Li, Anind Dey, and Jodi Forlizzi. 2010. A stagebased model of personal informatics systems. In Proceedings of the SIGCHI Conference on Human Factors in Computing Systems (CHI '10), 557-566. http://doi.acm.org/10.1145/1753326.1753409

13. Alessandro Marcengo, and Amon Rapp. 2013 Visualization of Human Behavior Data: The Quantified Self. In Innovative Approaches of Data Visualization and Visual Analytics, Mao Li Huang. and Weidong Huang (Eds.). IGI Global, Hershey, PA, 236-265.

14. Alessandro Micarelli, Fabio Gasparetti, Filippo Sciarrone, and Susan Gauch. Personalized search on the world wide web. In The Adaptive Web, Methods and Strategies of Web Personalization, Peter Brusilovsky, Alfred Kobsa, and Wolfgang Nejdl (Eds.). LNCS 4321. Springer-Verlag Berlin Heidelberg, 195-230.

15. Frank E. Ritter, Gordon D. Baxter, Gary Jones, and Richard M. Young. 2000. Supporting cognitive models as users. ACM Trans. Comput.-Hum. Interact. 7, 2, 141-173. http://doi.acm.org/10.1145/353485.353486

16. Bracha Shapira, Lior Rokach, and Shirley Freilikhman. 2013. Facebook single and cross domain data for recommendation systems. User Modeling and User-Adapted Interaction 23, 2-3, 211-247.

17. Esther Strauss, Elisabeth M. S. Sherman, Otfried Spreen. 2006. A Compendium of Neuropsychological Tests: Administration, Norms, and Commentary. Oxford University Press, US. 